\title{
A 4I year old woman with severe nausea and vomiting and known breast cancer with metastasis in the pelvis and femur
}

\section{Keywords: letrozole, hypercalcaemia, pyloris, ileus, vomitus Opinion}

This patient is relatively young with a breast cancer that has metastasis in the femur and pelvis. She was treated with radiotherapy and got letrozole, I assume that the tumour was receptor positive and reduction of estrogen production desirable. Despite this she develops severe nausea and vomiting.

My differential diagnosis is as follows:

i. Hypercalcaemia due to skeletal metastasis.

ii. Bowel obstruction due to metastasis in the GI tract, the pyloris is a known site that is at risk for breast cancer metastasis.

iii. Brain metastasis with increasing intracranial pressure.

iv. Liver failure or hepatitis.

When it is due to brain metastasis I would expect vomiting in the morning and head-ache and not so much vomiting. If there is an obstruction of the GI tract I would expect vomiting after feeding and depending on the site of the obstruction signs of an ileus with distended abdomen, high pitched bowel sounds, faecal vomitus and abdominal pain. My most likely diagnosis would be hypercalcaemia due to bony metastasis and this will explain the severe nausea as well as the vomiting. A simple laboratory test will confirm this or exclude it. She presents with severe lumbar spine pain and this would fit with progressive metastasis in the skeleton and perhaps a pathological compression fracture of L4 or L5. Clinically I can suspect that if she has percussion pain on these vertebrae and more pain when she is sitting or standing. In that case it is a nociceptive pain and I expect that it will respond well to opoids.

My management plan will depend if I want to treat her at home or in the hospital. At home I can take blood for $\mathrm{Ca} / \mathrm{P}$ and do a urine dipstick to exclude a kidney stone. If she is seen in OPD I will do a lumbar spine X-ray to see the extend of her metastatic disease. But if the patient is too weak and her condition is too poor for another course of palliative radiotherapy I will keep her at home and treat her on my clinical diagnosis with proper pain killers. In her case she has severe pain and I would go for morphine despite her nausea and use the subcutaneous route or rectal supps. Start with $10 \mathrm{mg} 6$ hourly and increase according to the response with an increase of $50 \%$ of the dose. In the home situation I will avoid IV morphine and drips. If this is a patient on medical aid Fentanyl patches will work also and are very effective and only need to be changed once every three days.

The nausea can be treated by lowering her serum calcium (iv fluids and bisphosphonates in case the life expectancy is longer than in this patient) and I would give her an anti-emetic drug. A cheap and readily available drug is metoclopramide, haloperidol, cyclizine
Volume 2 Issue 3 - 2018

\author{
Martin Bac \\ Family physician, Faculty of Health Sciences, University of \\ Pretoria, South Africa
}

Correspondence: Martin Bac, Family physician, Faculty of Health Sciences, University of Pretoria, South Africa, Tel +27795896688,Email martin.bac@up.ac.za

Received: April 17, 2018 | Published: May 15, 2018

or prochlorperazine per rectum or in the case of prochlorperazine by deep IM injection. In cancer patients who get chemotherapy the serotonin (5HT3) antagonists are also a good option and ondansetron can be given orally (melts on the tongue) or by IM injection. In this patient I would not start with this also because of the costs. Sometimes corticosteroids such as dexamethasone work very well especially if there is a raised intracranial pressure. Then the scenario is that the patient's disease is progressing rapidly probably due to widespread metastasis and the fact that she is unable to eat and drink well. She is diagnosed with terminal breast cancer and the prognosis is that she will succumb within a week. The family asks for a meeting to discuss admission in a hospice. I would find out what the reason is that they consider admission in a hospice. Are they afraid to see her mother die, are they not able to provide the care needed, there misconceptions about what is needed or can happen. Do they feel unsupported by the health team?

It is only thereafter that we can start to discuss what is your wife's wish? Does she want to stay at home or does she prefer to go to a hospice? Will she feel safer there perhaps? My task as a doctor will be to answer any questions they have and to help them to review the options they have. It is caring for her at home with support of the home based care team or community nurses or in some area's the hospice will have an outreach team. The second option is to go to the hospice and stay there till the end. If the family expects a long sick bed and that they cannot care for a long time for her I would give them my prognosis and say that I will be available for consultation in both situations. Can they care for her if it is only for a limited period of time? What are their needs at the moment and how can they is best met? I will involve another member of the palliative care team depending on the issues.

\section{Acknowledgements}

None.

\section{Conflict of interest}

The author declares no conflict of interest. 